\title{
CONTINUITY OF TRANSLATION AND SEPARABLE INVARIANT SUBSPACES OF BANACH SPACES ASSOCIATED TO LOCALLY COMPACT GROUPS
}

\author{
Colin Graham¹, Anthony To-Ming Lau² ${ }^{2}$, Michael Leinert ${ }^{3}$
}

\section{Introduction.}

Let $G$ be a locally compact group. How big can a separable ideal of the algebra of regular Borel measures on $G$ be? More generally, let $\Phi$ be a normed linear space. Assume that $G$ acts on $\Phi$ as linear isometries. Suppose that $\Psi$ is a $G$-invariant subspace of $\Phi$. How big is $\Psi$ ? A fairly elementary argument shows that under a very mild continuity assumption on the action of $G$, if $\Psi$ is separable, then $\Psi \subseteq \Phi_{C}$, where $\Phi_{C}$ denotes elements of $\Phi$ on which the operation of $G$ is continuous.

It is the purpose of this note to report on results we have obtained on separability of $\Psi$, the dimension of $\Phi_{c}$, and characterizations of the set $\Phi_{C}$ for various $\Psi$ and $\Phi$. Details and related results will appear in [GLL1] and [GLL2].

This work was motivated by a question raised by Professor Ryll-Nardewski and communicated to us by Professor Hartman: "Must a translation-invariant subspace of $M(G)$ that is not contained in $L^{1}(G)$ have dimension $c$ ?" We are grateful to them both.

\section{Lower semicontinuous representations.}

Let $G$ be a locally compact group. By a representation $T$ of $G$ on a normed linear space $\Phi$ we mean a mapping $x \rightarrow T(x)$ from $G$ into the group of linear isometries from $\Phi$ into $\Phi$ such that $T\left(x_{1} x_{2}\right)=T\left(x_{1}\right) \circ T\left(x_{2}\right), x_{1}, x_{2} \in G . T$ is said to be lower semicontinuous if for each $\mu \in \Phi$, and

1980 Mathematics Subject Classification (1985 Revision). 43A15.

${ }^{1}$ Research partially supported by grants from the NSF (USA) and NSERC (Canada).

2Research partially supported by a grant from NSERC (Canada).

3Part of this research done while the third author was visiting Northwestern University and the University of Alberta. 
each $\varepsilon>0$, the set

$$
\{x \in G:\|T(x) \mu-\mu\|>\varepsilon\}
$$

is open in $G$. Let $\Phi_{C}$ denote the set of all $\mu \in \Phi$ such that $x \rightarrow T(x) \mu$ is continuous from $G$ into $\Phi$ when $\Phi$ has the norm topology. The principal tool in this section is the following observation:

LEMMA 2.1. Let $G$ be a locally compact group and $T$ be a lower semicontinuous representation of $G$ on the normed space $\Phi$. Let $\mu \notin \Phi_{C}$. Then there exists $\varepsilon>0$ such that $H=$ $\{x \in G:\|T(x) \mu-\mu\|>\varepsilon\}$ is an open dense subset of $G$.

Lemma 2.1 yields the following generalization and improvement of the result of Larsen [L] and Tam $[\mathrm{T}]$ :

THEOREM 2.2. Let $G$ be a non-discrete locally compact group, and $T$ be a lower semi-continuous representation of $G$ as linear isometries on the normed space $\Phi$, and $\Psi$ a $G$-invariant subspace of $\Phi$. If $\Psi$ is separable, then $\Psi \subseteq \Phi_{c}$.

THEOREM 2.3. Let $G$ be a non-discrete locally compact group, and $T$ be a lower semi-continuous representation of $G$ as linear isometries on the normed space $\Phi$, and $\Psi$ a $G$-invariant subspace of $\Phi$. If $\Psi \not \subset \Phi{ }_{c}$, then $\Psi$ has dimension at least $c$ in the norm topology.

Let $M(G)$ denote the space of regular Borel measures on $G$ and $M_{a}(G)$ denote the closed ideal of measures in $M(G)$ absolutely continuous with respect to Haar measure.

\section{COROLlary 2.4. Let $G$ be a non-discrete locally compact group and $\Psi$ a left} translation-invariant subspace of $M(G)$. If $\Psi \not \subset M_{a}(G)$, then $\Psi$ has dimension at least $c$.

If $G$ is a locally compact abelian group, let $M_{0}(G)$ denote all measures in $M(G)$ whose Fourier transform vanishes at infinity. Let $\|\mu\|_{0}=\sup \{|\hat{\mu}(\chi)|: \chi \in \hat{G}\}$ where $\hat{G}$ denotes the dual group of $G, \mu \in M(G)$.

\section{COROLLARY 2.5. Let $G$ be a non-discrete locally compact group and $\Psi$ a left} translation-invariant subspace of $M(G)$. If $\Psi \not \subset M_{0}(G)$, then $\Psi$ has dimension at least $c$ in the $\|\cdot\|_{0}-$ norm topology.

For each $x \in G$, let $\delta_{x}$ denote the Dirac measure at $x$. By $\operatorname{Rad} M_{a}(\mathrm{G})$ we shall mean the intersection of all maximal ideals of $M(G)$ that are not contained in the set of ideals identified with the dual group $\hat{G}$ of $G$. Since we do not know whether or not the action of $G$ on $M(G)$ is 
lower semicontinuous when $G$ is abelian and $M(G)$ has the spectral radius norm (see problem 1 in section 6), we cannot apply Theorem 2.3 to prove:

THEOREM 2.6. Let $G$ be a non-discrete locally compact abelian group and $\Psi$ be a translation-invariant subspace of $M(G)$. If $\Psi \not \subset R a d M_{a}(G)$, then $\Psi$ has dimension at least $c$ in the spectral radius norm topology.

The proof of Theorem 2.6 depends on the following observation:

LEMMA 2.7. Let $\vee$ be a singular measure on a locally compact group $G$ and let $H=\{x \in G$ : $\left.\delta_{x} * v \perp v\right\}$. Then there exists a set $C$ of cardinality at least $c$ such that $x^{-1} y \notin H$ whenever $x$ and $y$ are distinct elements of $C$.

\section{Separable subspaces in $B(G)$.}

Let $G$ be a locally compact group and $P(G)$ be the set of continuous positive definite functions on $G$. Let $B(G)$ be the linear span of $P(G)$. Then $B(G)$ is an algebra under pointwise mulitiplication and invariant under left and right translations by elements of $G . B(G)$ can be identified as the continuous dual of $C^{*}(G)$, the enveloping $C^{*}$-algebra of $L^{1}(G)$, i.e. $\langle\varphi, f\rangle=$ $\int \varphi(t) f(t) d t$ for any $\varphi \in B(G), f \in L^{1}(G)$. Then $B(G)$, with the dual norm on $C^{*}(G)^{*}$, is a commutative Banach algebra, called the Fourier-Stieltjes algebra of $G$. Furthermore, if $G$ is abelian, then $B(G)$ is isometrically isomorphic to $M(\hat{G})$ by the Bochner's Theorem. Let $A(G)$ denote all elements in $B(G)$ of the form:

$$
\varphi(x)=\left\langle\ell_{x} h, k\right\rangle, \quad h, k \in L^{2}(G)
$$

$\ell_{x} h(y)=h\left(x^{-1} y\right), x, y \in G$. Then $A(G)$ is a closed ideal in $B(G)$ (called the Fourier algebra of $G$ ) isometrically isomorphic to $L^{1}(\hat{G})$ when $G$ is abelian. We refer the readers to [E] for more details about $A(G)$ and $B(G)$.

Let $E$ denote the weak*-closure of the extreme points of $P_{0}(G)=\{\varphi \in P(G): \varphi(e) \leq 1\}$. Then, when $G$ is abelian, $E \backslash\{0\}$ corresponds exactly to the characters on $G$. A subset $\Phi$ of $B(G)$ is invariant if $\varphi f \in \Phi$ for all $\varphi \in E$ and all $f \in \Phi$. Clearly, every ideal is invariant.

THEOREM 3.1. (i) Let $G$ be an amenable locally compact grup. Let $\Phi$ be an invariant separable subspace of $B(G)$. If $\phi \in \Phi$ and $\phi \neq 0$, then there exists $f \notin E$ such that $\phi f \in$ and $\phi f \neq 0$. In particular, if $G$ is abelian, then $\Phi \subseteq A(G)$. 
(ii) The "ax $+b "$ group contains a separable ideal $\psi \subseteq B(G)$ and $\psi \nsubseteq \subset A(G)$.

THeOREM 3.2. (i) Let $\Phi$ be a separable invariant subspace of $\left(B(\mathrm{G}),\|\cdot\|_{\infty}\right)$ where $\|\cdot\|_{\infty}$ denotes the supremum norm on $B(G)$. If $G$ is amenable, then for each $\phi \in \Phi, \phi \neq 0$, there exists $\gamma \in E$ such that $\gamma \phi \neq 0$ and $\gamma \phi \in C_{0}(G)$. In case that $G$ is abelian, that implies $\Phi \subseteq C_{0}(G)$.

(ii) If $G$ is either the Euclidean motion group or the $S L(2, \mathrm{R})$, then $\left(B(G),\|\cdot\|_{\infty}\right)$ is separable.

(iii) If $G$ is a [Moore]-group (i.e. each of its irreducible unitary representations is finite dimensional), and if $\left(B(G),\|\cdot\|_{\infty}\right)$ is separable, then $G$ is compact.

\section{Continuity of translation in $L^{\infty}(G) *$ and related subspaces.}

Let $G$ be a locally compact group and $L^{\infty}(G)$ be the space of essentially bounded complex measurable functions on $G$ with the essential sup norm. Let $W$ be a $C^{*}$-subalgebra of $L^{\infty}(G)$ containing constants and invariant under left translation $\ell_{a}, a \in G$, where $\left(\ell_{a} f\right)(x)=f\left(a^{-1} x\right), x \in$ G. A linear functional $m$ on $W$ is called left invariant mean if $m \geq 0,\|m\|=1$ and $m\left(\ell_{a} f\right)=$ $m(f)$ for all $a \in G, f \in W . G$ is amenable if $L^{\infty}(G)$ has a left invariant mean. As well known, all compact and all abelian groups are amenable. But any locally compact group $G$ containing the free group on two generators as a closed subgroup (i.e. $\operatorname{SL}(2, \mathbf{R})$ ) is not amenable. We refer the interested readers to the classic of Greenleaf [Gr] and the recent books of Pier [P] and Paterson $[\mathrm{Pa}]$.

Let $\left(W^{*}\right)_{C}$ denote all elements $\varphi$ in $W^{*}$ such that the map $G \rightarrow W^{*}, x \rightarrow \ell_{x}^{*} \varphi$ is continuous when $W^{*}$ has the norm topology. Then, obviously, $\left(W^{*}\right)_{C}$ contains the linear span of the set of left invariant means on $W$.

THEOREM 4.1. Let $G$ be a locally compact group. Then $G$ is compact if and only if for each $\mu \in\left(L^{\infty}(G)^{*}\right)_{c}$ there exists a left invariant mean $v$ such that $\mu<<$.

THEOREM 4.2. Let $G$ be a noncompact locally compact group. Let $W$ be a left translation-invariant $C^{*}$-subalgebra of $L^{\infty}(G)$ such that $W \cap C B(G)$ separates points and contains constant functions. Then there exists $\mu \in\left(W^{*}\right)_{c}$ such that $\mu$ is singular with respect to every left translation-invariant mean on $W$.

THEOREM 4.3. Let $G$ be a unimodular locally compact group with an infinite closed discrete subgroup $H$. Then there exists an element $\mu \in\left(L^{\infty}(G)^{*}\right)_{C}$ that is singular with respect to every 
translation-invariant mean on $G$ and with respect to $L^{1}(G)$.

THEOREM 4.4. Let $G$ be a locally compact group.

(i) $\left(L^{\infty}(G)^{*}\right)_{C}=L^{\infty}(G)^{*}$ if and only if $G$ is dsicrete.

(ii) $\left(L^{\infty}(G)^{*}\right)_{C}=L^{1}(G)$ if and only if $L^{\infty}(G)$ has a unique left invariant mean.

REMARK. If $G$ is amenable as a discrete group, then $L^{\infty}(G)$ has more than one left invariant mean (see $[\mathrm{Gn}]$ and $[\mathrm{R}]$ ). However, for $n \geq 3$, and for $G=S O(n, \mathbb{R})$, the situation is different: $L^{\infty}(G)$ has a unique left invariant mean (see [M] and [D]).

Let $\operatorname{LUC}(G)$ denote the space of bounded complex-valued left uniformly continuous function on $G$. Then, as well known, $L U C(G)=L^{\infty}(G){ }_{C}$ when $G$ acts on $L^{\infty}(G)$ by translation. It can be shown that $\left(L U C(G)^{*}\right)$ is an $L$-subspace of $L U C(G)^{*}$. However, $\left(L^{\infty}(G)^{*}\right)$ is not an $L$-space in general (e.g. when $G=\mathbf{R}$ ).

THEOREM 4.5. Let $G$ be a locally compact group. Then

(i) $\left(L U C(G)^{*}\right)_{C}=\operatorname{LUC}(G)^{*}$ if and only if $G$ is discrete.

(ii) $\left(L U C(G)^{*}\right)_{C}=L^{1}(G)$ if and only if $G$ is compact.

(iii) $\left(L U C(G)^{*}\right){ }_{C}$ contains a measure on $\Delta(L U C(G))$ with a non-zero part if and only if $G$ is discrete.

\section{Continuity of translation in $V N(G)$ and $V N(G) *$.}

Let $V N(G)$ denote the von Neumann algebra generated by left translations on $L^{2}(G)$. Then, as well known, $V N(G)$ coincides with the closure of $\left\{\rho(f): f \in L^{1}(G)\right\}$ in $\mathcal{B}\left(L^{2}(G)\right)$ in the weak operator topology where $\rho(f)(h)=f * h, h \in L^{2}(G)$. If $G$ is a locally compact abelian group, then $V N(G) \cong L^{\infty}(\hat{G})$. Furthermore, $A(G)$ can be identified as the unique predual of $V N(G)$ with $\langle\varphi, \rho(f)\rangle=\int \varphi(t) f(t) d t, f \in L^{1}(G)$, and $\|\varphi\|=\sup \left\{\left|\int \varphi(t) f(t) d t\right|:\|\rho(f)\| \leq 1\right\}$.

$G$ acts naturally on $V N(G)$ by the map $(x, T) \rightarrow \ell_{x} \circ T, x \in G, T \in V N(G)$ and $G$ acts on $V N(G)^{*}$ via the adjoint action.

THEOREM 5.1. Let $G$ be a locally compcat group.

(i) $V N(G)_{c}=V N(G)$ if and only if $G$ is discrete.

(ii) $V N(G){ }_{C}=C_{\lambda}^{*}(G)$ (the $C^{*}$-algebra generated by $\rho(f), f \in L^{1}(G)$ ) if and only if $G$ is compact. 
Let $G_{\mathrm{d}}$ denote the group $G$ with the discrete topology.

THEOREM 5.2. Let $G$ be a locally compact group. Suppose that $\left(V N(G)^{*}\right)_{C}=A(G)$. Then the following hold:

(i) If $G$ is amenable, then $G$ is compact.

(ii) If $G_{\mathrm{d}}$ is amenable, then $G$ is finite.

Note that in Theorems 4.4, 4.5, and 5.1, the first part is rather easy.

\section{Some open problems.}

1. Let $G$ be a locally compact abelian group. Is the action of $G$ on $M(G)$ defined by $(x, \mu) \rightarrow$ $\delta_{x} * \mu, x \in G, \mu \in M(G)$ lower semicontinuous when $M(G)$ has the spectral radius norm?

2. Can the amenability assumption be dropped in Theorem 3.1(i) and Theorem 3.2(i) ? Note that "amenability" in both Theorems 3.1(i) and 3.2(i) can be replaced by the following weaker condition which holds for all amenable groups, all free groups, and the group $\operatorname{SL}(2, \mathrm{R})$ (see $[\mathrm{CH}]$ ): there is a net in $A(G)$ with multiplier norm bounded by 1 and which converges to the constant one function in the weak*-topology.

3. Let $G$ be a non-discrete locally compact group. Does there always exist an element $\mu \epsilon$ $\left(L^{\infty}(G)^{*}\right){ }_{C}$ which is singular with respect to every translation-invariant mean on $G$ and with respect to $L^{1}(G)$ (see Theorem 4.3 ).

4. Let $G$ be a locally compact group such that $\left(V N\left(G^{*}\right)\right){ }_{C}=A(G)$. Is $G$ necessarily finite? (see Theorem 5.2).

5. If $\left(L^{\infty}(G)^{*}\right)$ is an $L$-space, is $G$ necessarily discrete?

\section{REFERENCES}

[CH] de Canniere, Jean and Haagerup, Uffe, Multipliers of the Fourier algebras of some simple Lie groups and their discrete subgroups, Amer. J. of Math. 107 (1985), 455-500.

[D] Drinfel'd, V.G., Finitely additive measure on $S^{2}, S^{3}$, invariant with respect to rotation, Functional Analysis and its Applications 18 (1984), 245-6 [Original Russian version in int Funck. Analic. i ego Priloz. 18 No. 3, July-Sept. 1984, p. 77.] 
[E] Eymard, P., L'algèbre de Fourier d'une groupe localement compact, Bull. Soc. Math. France 92 (1964), 181-236.

[GLL1] Graham, C.C. Lau, A.T.-M. and Leinert, M., Separable translation-invariant subspaces of $M(G)$ and other dual spaces on locally compact groups, Colloquium Mathematicum (to appear).

[GLL2] Graham, C.C. Lau, A.T.-M. and Leinert, M., Continuity of translation in the dual of $L^{\infty}(G)$ and related spaces (to appear).

[Gn] Granirer, E.E., Criteria for compactness and for discreteness of locally compact groups, Proceedings of the Amer. Math. Soc. 40 (1973), 615-624.

[Gr] Greenleaf, F.P., Invariant means on topological groups, Van Nostrand, Princeton, N.J., 1969.

[M] Margulis, G.A., Some remarks on invariant means, Monatsheft fur Mathematik 90 (1980), 233-235.

[P] Pier, J.-P., Amenable locally compact groups, Wiley, New York, 1984.

[Pa] Paterson, A., Amenability, A.M.S. mathematical surveys and monograph series, no. 29,1988 .

[R] Rudin, W., Invariant means on $L^{\infty}(G)$, Studia Mathematicum 44 (1972), 219-227.

[T] Tam, K.W., On measures with separable orbit, Proceedings Amer. Math. Soc. 23 (1969), 409-411.

Department of Mathematics

Northwestern University

Evanston, Illinois 60208-2925 USA

Department of Mathematics

University of Alberta

Edmonton, Alberta T6G 2G1 Canada

Institut fur Angewandte Mathematik

Universitat Heidelberg

Im Neuenheimer Feld

Gebäude 294

6900 Heidelberg 1, Germany 